\title{
The Protective and Recovery Effects of Fish Oil Supplementation on Cedar Pollen-Induced Allergic Reactions in Mice
}

\author{
Akiko Hirao $^{1,2^{*}}$, Naoki Furutani ${ }^{1 *}$, Hiroki Nagahama ${ }^{1}$, Misa Itokawa ${ }^{1}$, Shigenobu Shibata ${ }^{1}$ \\ ${ }^{1}$ Laboratory of Physiology and Pharmacology, School of Advanced Science and Engineering, Waseda University, Tokyo, Japan; \\ ${ }^{2}$ Japan Society for the Promotion of Science, Tokyo, Japan. \\ Email: blooming-cherry@moegi.waseda.jp
}

Received September $15^{\text {th }}, 2011$; revised November $2^{\text {nd }}, 2011$; accepted November $10^{\text {th }}, 2011$

\begin{abstract}
Obesity is associated with the production of pro-inflammatory cytokines and therefore can lead to worsening of allergic reactions. Thus, there is cross-talk between obesity and allergic reactions. In this study, we investigated whether the anti-obesity action of fish oil supplementation is involved in the anti-allergic action also induced by fish oil. We observed attenuation of cedar pollen-induced IgE serum increases in two experimental protocols: $15 \%$ fish oil supplementation for 8 weeks, which attenuated body weight increases compared with 15\% soybean supplementation, and 4\% fish oil supplementation for 2 weeks, which did not affect body weight increases compared with $4 \%$ soybean or $4 \%$ lard supplementation. The former but not the latter protocol attenuated sneezing after pollen challenge. Gene expression of TNF- $\alpha$ and IL-13, a Th2 cytokine, was moderately reduced in the trachea and nasal mucosa in mice fed fish oil supplements. In a third protocol, fish oil was administered for up to 15 weeks after a cedar pollen sensitization and challenge-induced increase in IgE levels. These levels decreased following fish oil supplementation but were almost unaffected by soybean oil supplementation. Surprisingly, the IL-13 gene expression level in the nasal mucosa and trachea was higher in the fish oil group than in the soybean oil group. These results suggest that fish oil supplementation before, during, or after antigen sensitization and challenge in mice helped to reduce cedar pollen-induced allergic reactions independent of its anti-obesity action. Fish oil supplementation can also exhibit pro- or anti-inflammatory action against IL-13 expression depending on the experimental protocol.
\end{abstract}

Keywords: Cytokine; Fish Oil; IgE; Obesity; Pollen Allergy

\section{Introduction}

Recent studies have shown that obesity gives rise to a state of chronic low-grade inflammation characterized by inflamed adipose tissue with increased infiltration of macrophages that produce pro-inflammatory cytokines $[1,2]$. These cytokines, such as TNF- $\alpha$, directly reduce insulin sensitivity through the insulin signaling pathway [3]. Weight loss and decreased fat deposition are ob- served in mice fed a diet containing high concentrations of the n-3 polyunsaturated fatty acids (PUFAs) docosa- hexaenoic acid (DHA) and eicosapentaenoic acid (EPA) [4]. n-3 PUFAs exert anti-inflammatory effects by reduc- ing pro-inflammatory cytokine expression in many chro- nic inflammatory conditions.

As fish oil is a good source of DHA and EPA, it may offer a useful anti-inflammatory strategy to decrease obesity and obesity-related disease in combination with

\footnotetext{
${ }^{*}$ These authors contributed equally to the manuscript.
}

other n-3 PUFAs [5].

Asthma is a major public health problem. Although medication and environmental manipulation play an important role in its treatment, dietary intervention appears to be an alternative therapy. Evidence from some epidemiological studies has suggested that consumption of n-3 PUFAs such as EPA and DHA may reduce the incidence of asthma, while conversely, a high-fat diet rich in $n-6$ fatty acids is associated with a higher risk of asthma [6-8]. However, clinical data from interventional studies on the effects of fish oil intake have been equivocal and controversial $[9,10]$. Some, but not all, studies observed clinical improvements in asthmatic symptoms following n-3 PUFA supplementation [11-14]. DHA and EPA enhance TNF- $\alpha$ and IL-6 secretion in macrophages [15-17], and dietary fish oil supplementation increases serum $\mathrm{TNF}-\alpha$ concentration in response to endotoxin challenges [15]; thus, n-3 PUFAs are regarded as pro-inflammatory factors. Treatment with n-3 PUFAs also increases TNF- $\alpha$, 
IL1a, and IL-6 expression in adipocytes, keratinocytes, splenocytes, and hepatocytes [18-22].

Recently, it was reported that dietary supplementation with n-3 PUFA-containing fish oil enhances the inflamematory cytokine response in a mouse model of ovalbumin (OVA)-induced allergic lung inflammation. On the other hand, numerous anti- inflammatory effects of n-3 PUFAs have also been reported, which mainly result from suppression of NF-kB [23]. In monocytes, n-3 PUFAs inhibit LPS-induced cytokine expression, including expression of IL-1b, IL-6, and TNF- $\alpha[24,25]$. Interestingly, recent research has shown that $n-3 / n-6$ PUFA levels are significantly lower in patients with grass pollen-induced allergic asthma than in healthy controls, and there is a tendency for higher bronchial hyperreactivity in patients with asthma [26]. Thus, fish oil may protect not only against obesity but also against polleninduced allergic inflammatory action through a reduction in pro-inflammatory cytokines.

In the present experiments, we examined whether fish oil supplementation develops anti- or pro-inflammatory responses in a mouse cedar pollen-induced allergy model. To clarify whether the anti-obesity action of fish oil supplementation is effective as an anti-allergic action against cedar pollen, we provided long-term fish oil supplementation to mice exhibiting anti-obesity action. Supplementation was performed before (protection) or after (recovery) sensitization and challenge with pollen to determine the protective or recovery effects of fish oil.

\section{Materials and Methods}

\subsection{Animals}

Five- or six-week-old female Balb/c mice (Tokyo Laboratory Animals, Tokyo, Japan) were housed at a temperature of $22^{\circ} \mathrm{C} \pm 2^{\circ} \mathrm{C}$ and humidity of $60 \% \pm 5 \%$ under a 12-h light/12-h dark (LD) cycle (lights on from 0800 to 2000). Zeitgeber time 0 (ZT0) was designated as lightson time and ZT12 as lights-off time under the LD cycles. The mice were fed a normal commercial diet (catalog \# MF; Oriental Yeast Co. Ltd., Tokyo, Japan) and given water ad libitum prior to the experiment. Animal care was conducted with permission from the Committee for Animal Experimentation of the School of Science and Engineering at Waseda University, Tokyo, Japan (permission \# 09A11) and in accordance with the law (No. 105 ) and notification (No. 6) of the Japanese Government.

\subsection{Study Protocol}

The standard mouse diet was AIN-93M formula (Oriental Yeast Co.), composed of $14 \%$ casein, $0.3 \%$ L-cysteine, $15 \%$ alpha corn starch, $47 \%$ beta corn starch, $10 \%$ su- crose, $4 \%$ soybean oil, $5 \%$ cellulose, $3.5 \%$ AIN-93 mineral mixture, $1 \%$ AIN-93 vitamin mixture, $0.25 \%$ bitartrate choline, and $0.0008 \%$ tert-butyl groups.

Three protocols were designed to compare the effects of different volumes of fish oil supplementation on the inflammatory response. In the first protocol, $4 \%$ soybean oil from the diet was substituted with $15 \%$ soybean oil or $15 \%$ menhaden oil (Sigma-Aldrich, St. Louis, MO, USA) and fed to 5- or 6-week-old mice for 3 weeks before and during sensitization and challenge with pollen.

For sensitization, mice were intraperitoneally injected with $0.1 \mathrm{~mL}(1 \mu \mathrm{g})$ of cedar pollen (Cryj 1, Hayashibara Biochemical Laboratories, Okayama, Japan) complexed with $20 \mathrm{mg} \mathrm{Al}(\mathrm{OH})_{3}$ on days 0 and 4 . On days $11-16$, the mice were challenged by exposure to cedar pollen solution. Five microliters of cedar pollen solution (100 $\mu \mathrm{g} / \mathrm{mL}$ diluted in phosphate-buffed saline) was poured into the nasal cavity of each mouse. Immediately after the last challenge on day 16 , we observed sneezing behavior for $15 \mathrm{~min}$ and then the mice were sacrificed under ether anesthesia.

The second protocol was identical to the first, but $4 \%$ soybean oil was substituted with $4 \%$ menhaden oil, $4 \%$ lard, or the standard diet (4\% soybean) without substitution.

In the third protocol, the same diet as the second protocol was fed to 6-week old-mice for 15 weeks before performing the same sensitization and challenge procedure as before. On weeks 1, 2, 4, and 9, blood samples were collected by retro- orbital bleeding under ether anesthesia, and mice were sacrificed on week 15. Plasma was separated by centrifugation at $4^{\circ} \mathrm{C}$, and samples were stored at $-80^{\circ} \mathrm{C}$ until analysis. The nasal mucosa was scratched, and the trachea was dissected. These tissues were dissolved in ISOGEN reagent (Nippon Gene, Tokyo, Japan), and total RNA was isolated.

\subsection{RNA Isolation and Real-Time RT-PCR}

A 50 ng aliquot of total RNA was reverse transcribed and amplified using the One-Step SYBR RT-PCR kit (Takara, Otsu, Japan) and an iCycler (BioRad, Hercules, CA, USA). Specific primer pairs were designed using Primer3 Input (version 0.4.0, [27]) on the basis of the published databases of Gapdh, Il-13, and Tnf-a genes: Gapdh, 5'-TGGTGAAGGTCGGTGTGAAC-3' (forward) and 5'-AATGAAGGGGTCGTTGATGG-3' (reverse); $1 l-13$, 5'-ATGGCCTCTGTAACCGCAAG-3' (forward) and 5'-GGCGAAACAGTTGCTTTGTG-3' (reverse); and Tnf- $a, 5^{\prime}$-ATCCGCGACGTGGAACTG-3' (forward) and 5'-ACCGCCTGGAGTTCTGGAA-3' (reverse). RT-PCR was performed under the following conditions, which were the same as described previously [28]: cDNA syn- 
thesis at $42^{\circ} \mathrm{C}$ for 15 min followed by $95^{\circ} \mathrm{C}$ for $2 \mathrm{~min}$, PCR amplification for 40 cycles with denaturation at $95^{\circ} \mathrm{C}$ for $5 \mathrm{~s}$, and annealing and extension at $60^{\circ} \mathrm{C}$ for 20 s. Gene amplification specificity was confirmed by measuring the size and purity of the PCR product by gel electrophoresis. The relative light unit of the PCR product of the target gene was normalized to that of Gapdh. Melt curve analysis was then performed.

\subsection{Blood IgE Measurements}

To measure IgE levels in mouse blood, we used commercially available mouse IgE ELISA kits (Shibayagi, Gunma, Japan) according to the manufacturer's protocol.

\subsection{Statistical Analysis}

Values are expressed as mean \pm SEM. One- or two-way analysis of variance (ANOVA) was performed using StatView software (SAS Institute, Cary, NC, USA) for statistical analysis, and post hoc analysis was performed using Student's t-test or Fisher's PLSD test. P $\leq 0.05$ was considered statistically significant.

\section{Results}

\subsection{Fish Oil Supplementation Had a Protective Effect on Cedar Pollen-Induced Allergic Reactions in Mice, Which Accompanied Its Anti-Obesity Action}

We observed that the fish oil-containing mouse chow diet significantly attenuated body weight gain (Figure 1(b)) as well as visceral fat percentage (Figure 1(c)) compared with the soybean oil-containing diet. The number of sneezes over the 15-min period following the last pollen challenge on day 16 was lower in mice receiving fish oil supplementation than in mice receiving soybean oil supplementation (Figure 1(c)). Moreover, blood IgE levels on day 16 were significantly lower in mice receiving fish oil than in mice receiving soybean oil (Figure 1(d)). Although no significant differences were observed in IL-13 or TNF- $\alpha$ gene expression levels in the nasal mucosa between the two groups (Figure 2(a)), the levels were reduced in the trachea of mice fed the fish oil-containing diet than in mice fed the soybean oil-containing diet (Figure 2(b)).

\subsection{Fish Oil Supplementation Had a Protective Effect on Cedar Pollen-Induced Allergic Reactions in Mice without an Anti-Obesity Action}

In the second experimental protocol, no differences in body weight were observed among mice fed the diet con- taining fish oil, soybean oil, or lard (Figure 3(b)), and the number of sneezes did not differ among the three groups (Figure 3(c)). Blood IgE levels were highest in mice fed the lard-containing diet, moderate in mice fed the soybean oil-containing diet, and lowest in mice fed the fish oil-containing diet; a significant difference $(\mathrm{P}<$ 0.05 , Fisher's PLSD test) was observed between IgE levels in mice fed the fish oil-containing diet and those fed the lard- containing diet (Figure 3(d)). IL-13 and TNF- $\alpha$ gene expression in the nasal mucosa did not differ among the three groups (Figure 4(a)). However, IL-13 $(\mathrm{P}>0.05)$ and TNF- $\alpha$ gene expression $(\mathrm{P}<0.05$, Fisher's PLSD test) reduced in the trachea of mice fed the fish

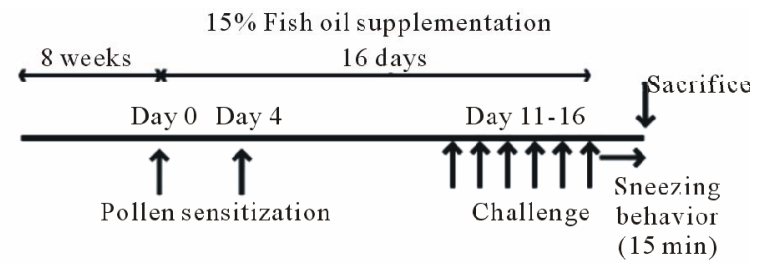

(a)

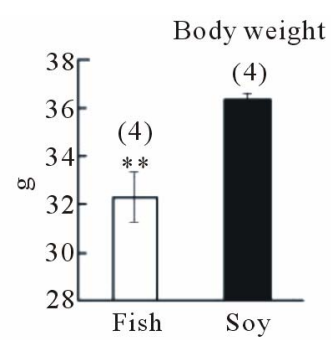

(b)

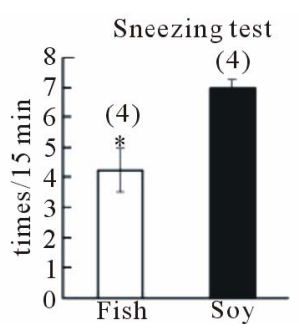

(d)

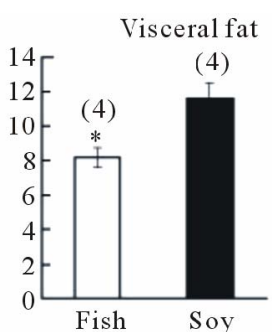

(c)

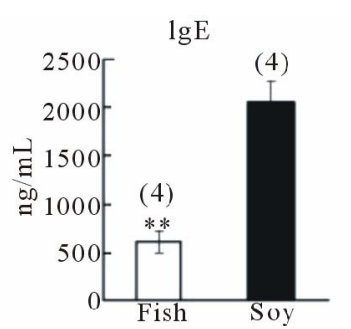

(e)
Figure 1. Effect of fish oil supplementation before and during sensitization and challenge with cedar pollen on body weight, visceral fat weight, IgE levels, and sneezing behavior in mice. (a) Experimental schedules. Five-weekold female mice were divided into two groups on the basis of the type of supplement provided: $15 \%$ fish oil supplementation and $15 \%$ soybean oil supplementation; (b) and (c) Body weight and visceral fat weight at the time of sacrifice. Fish oil supplementation significantly attenuated body weight and visceral fat weight increases; (d) Number of sneezes during a 15-min period after the last challenge with cedar pollen; (e) Serum IgE levels after the last challenge on day 16. Data are presented as mean \pm SEM (fish oil, $n=4$; soybean oil, $n=4)$. ${ }^{* *} P<0.01,{ }^{*} P<0.05$ vs soybean oil group (Student's t-test). 

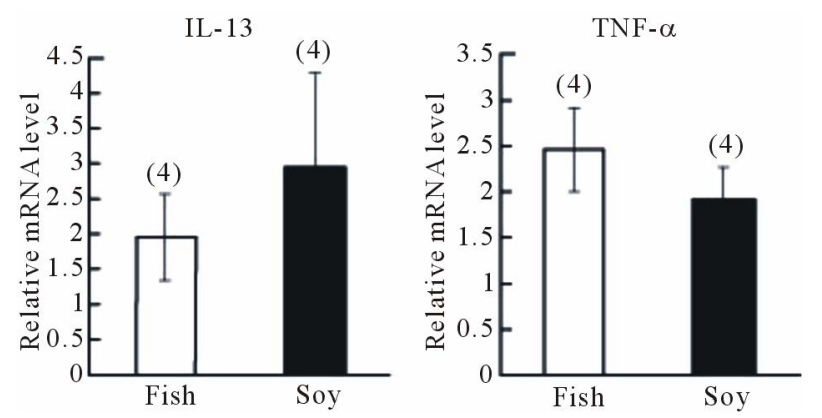

(a)
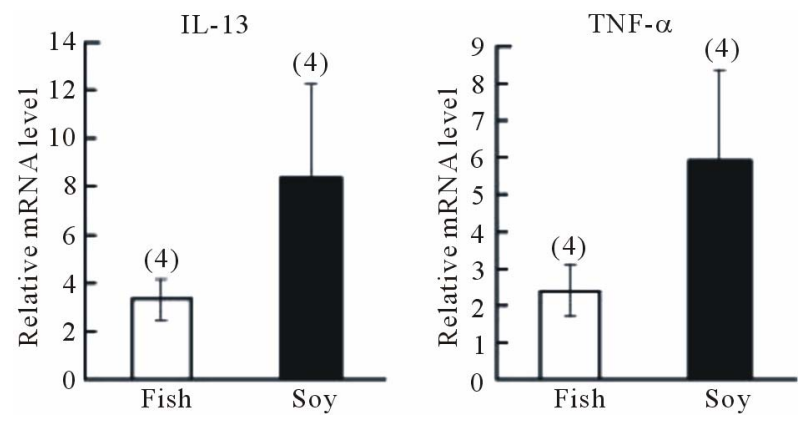

(b)

Figure 2. Effect of fish oil supplementation before and during sensitization and challenge with cedar pollen on gene expression of IL-13 and TNF- $\alpha$ in the nasal mucosa and trachea of mice. (a) IL-13 and TNF- $\alpha$ mRNA levels in the nasal mucosa; (b) IL-13 and TNF- $\alpha$ mRNA levels in the trachea. The relative levels of each data set are normalized to the corresponding GAPDH RNA levels. Data are presented as mean \pm SEM (fish oil, $n=4$; soybean oil, $n=4$ ).

oil-containing diet than of mice fed the lardcontaining diet (Figure 2(b)).

\subsection{Fish Oil Supplementation Had a Recovery Effect on Cedar Pollen-Induced Allergic Reactions in Mice}

We next examined whether fish oil supplementation could recover an allergic reaction already established by cedar pollen. At week 0 , all mice were fed the diet containing $4 \%$ soybean oil; therefore, no initial difference was observed in $\operatorname{IgE}$ levels between the two groups (Figure 5(b)). After changing the mouse diet from soy bean oil to fish oil in one group, $\operatorname{IgE}$ levels decreased significantly following the recovery periods $(\mathrm{P}<0.05$, recovery time, one-way ANOVA). IgE levels remained almost constant for 15 weeks in mice fed the soybean oilcontaining diet. Surprisingly, IL-13 mRNA expression levels in the nasal mucosa $(\mathrm{P}<0.05$, Fisher's PLSD test) and trachea were higher in the fish oil group than in the soybean oil group. TNF- $\alpha$ gene expression was similar between the two groups. The body weight of mice fed fish oil for 15 weeks was slightly lower $(23.3 \pm 0.33 \mathrm{~g}, \mathrm{n}=3)$

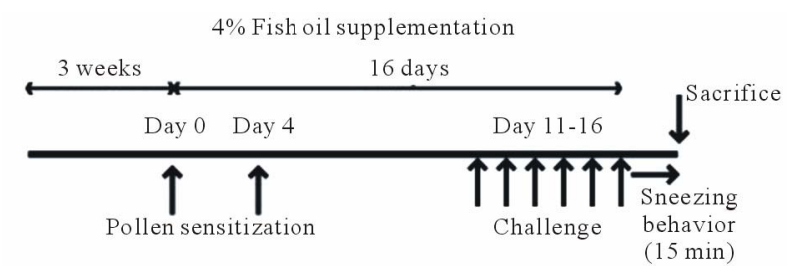

(a) (b)

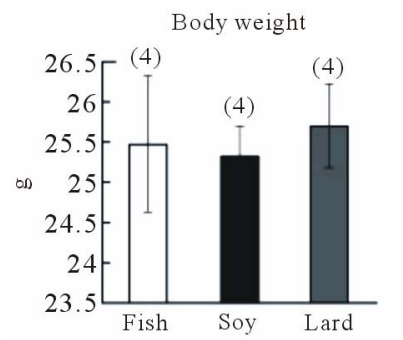

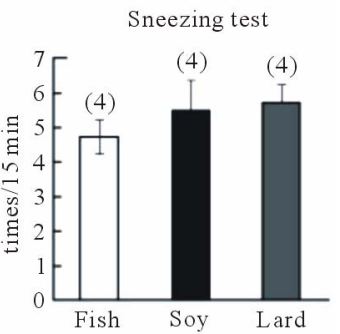

(c)

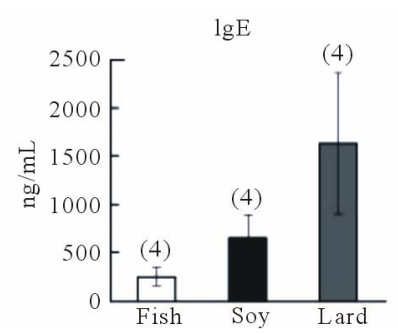

(d)

Figure 3. Effect of fish oil supplementation before and during sensitization and challenge with cedar pollen on body weight, IgE levels, and sneezing behavior in mice. (a) Experimental schedules. Five-week-old female mice were divided into three groups on the basis of the type of supplement provided: $4 \%$ fish oil supplementation, $4 \%$ soybean oil supplementation, and $4 \%$ lard supplementation; (b) Body weight at the time of sacrifice. No differences in body weight were observed among the three groups; (c) Number of sneezes during a 150 -min period after the last challenge with cedar pollen. Moreover, since there was much number of times of sneezes, the rate in every 15 minutes was shown in the graph; (d) Serum IgE levels after the last challenge on day 16. Data are presented as mean \pm SEM (fish oil, $n=$ 4; soybean oil, $n=4$; lard, $n=4$ ).

than that of mice fed soybean oil $(23.7 \pm 0.43 \mathrm{~g}, \mathrm{n}=4)$.

\section{Discussion}

In this study, supplementing mouse chow diet with fish oil had both anti-allergic and anti- obesity actions. Two different feeding protocols showed that inclusion of fish oil in the diet of mice reduced the cedar pollen-induced increase in blood IgE levels. The high concentration $(15 \%)$ and long-lasting (8 weeks) supplementation of fish oil also reduced the observed increase in body weight and visceral fat. Fish oil treatment has previously been 

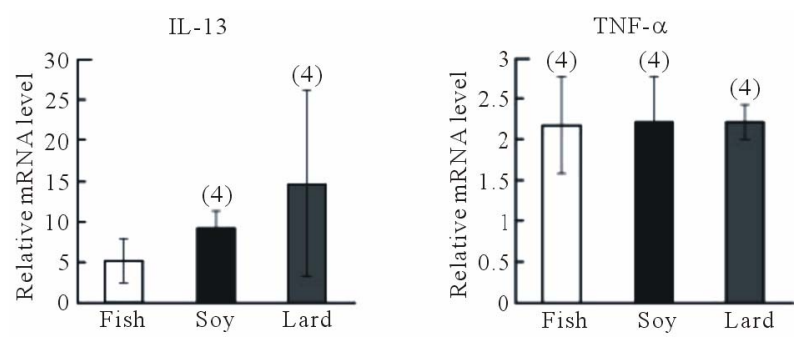

(a)

\section{IL-13}

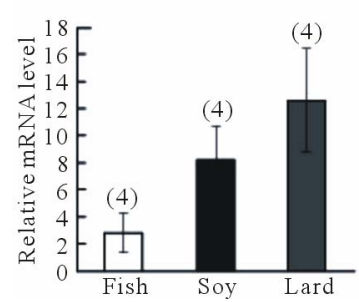

(b)

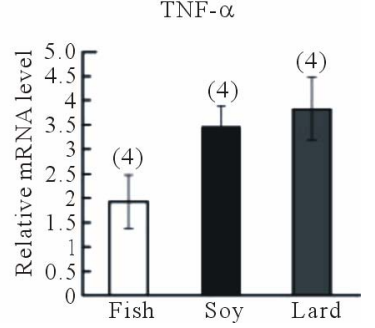

Figure 4. Effect of fish oil supplementation before and during sensitization and challenge with cedar pollen on gene expression of IL-13 and TNF- $\alpha$ in the nasal mucosa and trachea of mice. (a) IL-13 and TNF- $\alpha$ mRNA levels in the nasal mucosa; (b) IL-13 and TNF- $\alpha$ mRNA levels in the trachea. The relative levels of each data set are normalized to the corresponding GAPDH RNA levels. Data are presented as mean \pm SEM (fish oil, $n=4$; soybean oil, $n=4$; lard, $n=4)$. "P $<0.05$ vs soybean oil group (Student's $t-$ test).

shown to effectively lower blood lipid levels in patients with hypertriglyceridemia [29,30] and prevent abdominal fat accumulation compared with other types of dietary oils [31-33]. It is well known that obesity exacerbates allergic reactions such as asthma [34], which is why fish oil-induced anti-obesity actions may have been involved in the reduction of IgE levels in the present study. To clarify this possibility, we examined the effect of $4 \%$ fish oil on pollen-induced IgE increases in mice with similar body weights compared with $4 \%$ soybean oil or $4 \%$ lard. As a result, body weight was almost identical among the three groups of mice, most probably because the oil component of the chow diet was small (4\%) and the feeding periods were short ( 2 weeks). Nevertheless, we again found that fish oil supplementation reduced pollen-induced IgE levels, strongly suggesting that fish oil has protective effects on pollen-induced allergic reactions independent of its anti-obesity action. Using cedar pollen as the antigen, we investigated the sneezing behavior of mice following pollen challenge by counting the number of sneezes. In the first experiment, fish oil supplementation significantly reduced the number of sneezes, but no statistical difference was observed in the second experiment between mice fed the diet containing

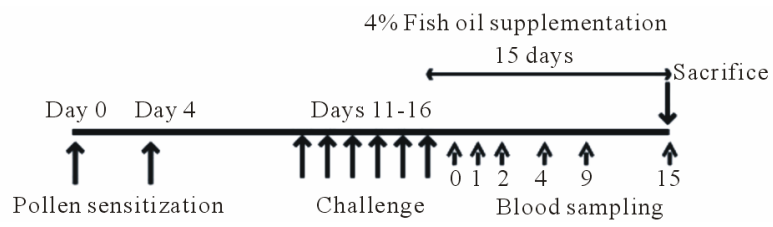

(a)

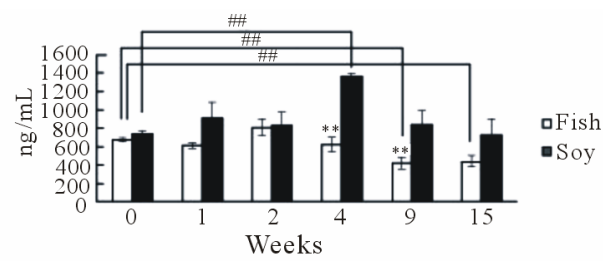

(b)

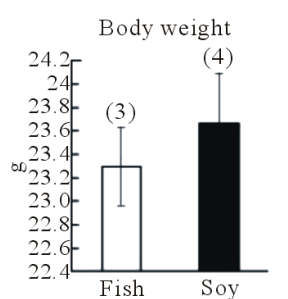

(c)
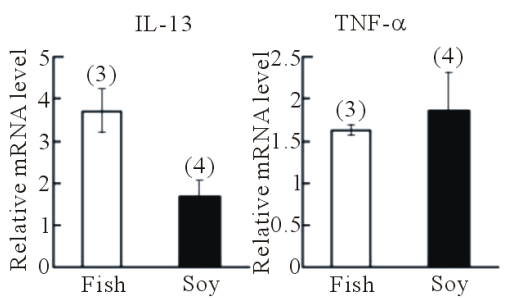

(d)

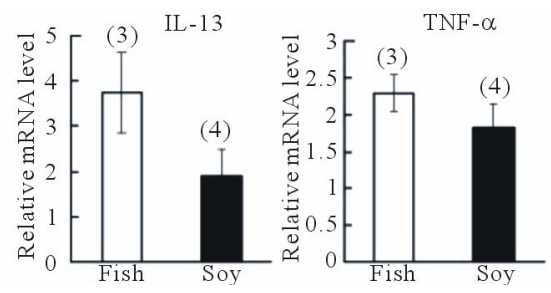

(e)

Figure 5. Effect of fish oil supplementation after sensitization and challenge with cedar pollen on serum IgE levels and gene expression of IL-13 and TNF- $\alpha$ in the nasal mucosa and trachea of mice. (a) Experimental schedules. Six-week-old female mice were exposed to cedar pollen. This induced allergy. After the last challenge with cedar pollen on day 16, mice were divided into two groups on the basis of the type of supplement provided: $4 \%$ fish oil supplementation and $4 \%$ soybean oil supplementation. Blood was collected to measure serum IgE levels on weeks $0,1,2$, 4, 9, and 15; (b) The time course of IgE levels after fish oil or soybean oil supplementation; (c) Body weight at the time of sacrifice. No differences in body weight were observed between the two groups; (d) IL-13 and TNF- $\alpha$ mRNA levels in the nasal mucosa; (e) IL-13 and TNF- $\alpha$ mRNA levels in the trachea. The relative levels of each data set are normalized to the corresponding GAPDH RNA levels. Data are presented as mean \pm SEM (fish oil, $n=3$; soybean oil, $n=4$ ). ${ }^{* *} \mathbf{P}<0.01,{ }^{*} \mathbf{P}<0.05$ vs soybean oil group (Student's t-test). ${ }^{\# \#} \mathrm{P}<0.01$ vs week 0 (Fisher's PLSD test).

fish oil, soybean oil, or lard. Fish oil supplementation was performed for longer and at higher concentrations in the first than in the second experiment, which is consistent 
with previous findings that at least 2 weeks are needed to replace arachidonic acid by n-3 PUFAs [35]. Thus, higher concentrations of fish oil may be necessary to effect an anti-allergic action against behaviors such as sneezing.

Blood IgE levels increased following cedar pollen challenge through Th2 responses, and mRNA expression levels of the Th2-specific cytokine IL-13 decreased, albeit non-significantly, in both the nasal mucosa and trachea in the fish oil group. mRNA levels of the general inflammatory cytokine TNF- $\alpha$ were reduced in the trachea but unaffected in the nasal mucosa following fish oil supplementation. This noticeable effect in the trachea suggests that fish oil supplementation may reduce cedar pollen-induced asthma. In clinical experiments, it was recently reported that n-3/n-6 PUFA levels were signifycantly lower in patients with grass pollen-induced allergic asthma than in healthy controls and that these patients showed a higher risk of bronchial hyperreactivity [26]. On the other hand, fish oil supplementation enhances the production of the pro-inflammatory cytokines IL-5 and IL13 in mouse lung tissue following OVA challenge [35].

This discrepancy with the present findings could reflect differences in the protocols because we administered fish oil supplementation both before and after sensitization and challenge with cedar pollen. We administered fish oil for up to 15 weeks after sensitization and challenge with cedar pollen in mice to investigate whether fish oil supplementation could recover the allergic response to cedar pollen. After the 15-week period, IgE levels slowly decreased to reach approximately half their initial level compared with almost stable IgE levels following soybean oil supplementation. In contrast, IL-13 mRNA expression levels were higher in the fish oil group than in the soybean oil group. Considering this result and the findings of Yin et al. (2009), we suggest that fish oil supplementation has protective effects on antigen-induced IL-13 production but provides weak therapeutic and recovery effects. In summary, we found that fish oil supplementation both before and after antigen sensitization and challenge reduced allergic responses such as IgE production induced by cedar pollen. Gene expression levels of the allergic cytokines IL-13 and TNF- $\alpha$ decreased in mice supplemented with fish oil supplementation before sensitization and challenge and increased in mice supplemented with fish oil supplementation after sensitization and challenge. Therefore, human consumption of fish oil may help reduce the symptoms of cedar pollen- induced allergic reactions.

\section{Acknowledgements}

This work was partially supported by a Grant-in-Aid for JSPS Fellows to A. H. and by Grants-in-Aid for Scientific Research $(23300278,23659126)$ as well as from the
Fuji Foundation for Protein Research (2010) and the Iijima Memorial Foundation for the Promotion of Food Science and Technology (2011) to S.S.s, and part of the outcome of research performed under a Waseda University Grant for Special Research Projects (Project number: 2010A-047).

\section{REFERENCES}

[1] H. Y. Xu, Glenn T. Barnes, Q. Yang, G. Tan, D. S. Yang, C. J. Chou, J. Sole, A. Nichols, J. S. Ross, L. A. Tartaglia and H. Chen, "Chronic Inflammation in Fat Plays a Crucial Role in the Development of Obesi Obesity-Related Insulin Resistance," Journal of Clinical Investigationt, Vol. 112, No. 12, 2003, pp. 1821-1830. doi:10.1172/JCI19451

[2] S. P. Weisberg, D. McCann, M. Desai, M. Rosenbaum, R. L. Leibel and A. W. Ferrante Jr., "Obesity is Associated with Macrophage Accumulation in Adipose Tissue," Journal of Clinical Investigationt, Vol. 112, No. 12, 2003, pp. 1796-1808. doi:10.1172/JCI200319246

[3] P. Peraldi, G. S. Hotamisligil, W. A. Buurman, M. F. White and B. M. Spiegelman, "Tumor Necrosis Factor (TNF)Alpha Inhibits Insulin Signaling through Stimulation of the p55 TNF Receptor and Activation of Sphingomyelinase," The Journal of Biological Chemistry, Vol. 271, No. 22, 1996, pp. 13018-13022.doi:10.1074/jbc.271.22.13018

[4] J. Ruzickova, M. Rossmeisl, T.Prazak, P. Flachs, J. Sponarova, M. Vecka, E. Tvrzicka, M. Bryhn and J. Kopecky, "Omega-3 PUFA of Marine Origin Limit Diet-Induced Obesity in Mice by Reducing Cellularity of Adipose Tissue," Lipids, Vol. 39, No. 12, 2004, pp. 1177-1185. doi:10.1007/s11745-004-1345-9

[5] L. M. Browning, "n-3 Polyunsaturated Fatty Acids, Inflammation and Obesity-Related Disease," Proceedings of the Nutrition Society, Vol. 62, No. 2, 2003, pp. $447-$ 453. doi:10.1079/PNS2003252

[6] S. Mihrshahi, J. K. Peat, K. Webb, W. Oddy, G. B. Marks and C. M. Mellis, "Effect of Omega-3 Fatty Acid Concentrations in Plasma on Symptoms of Asthma at 18 Months of Age," Pediatric Allergy and Immunology, Vol. 15 , No. 6, 2004, pp. 517-522. doi:10.1111/j.1399-3038.2004.00187.x

[7] K. F. Chung, "Evaluation of Selective Prostaglandin E2 (PGE2) Receptor Agonists as Therapeutic Agents for the Treatment of Asthma," Science's STKE, Vol. 2005, No. 303, 2005, p. e47. doi:10.1126/stke.3032005pe47

[8] T. D. Mickleborough and K. W. Rundell, "Dietary Polyunsaturated Fatty Acids in Asthma- and Exerciseinduced Bronchoconstriction," European Journal of Clinical Nutrition, Vol. 59, No. 12, 2005, pp. 1335-1346. doi:10.1038/sj.ejcn.1602250

[9] S. L. Prescott and P. C. Calder, "n-3 Polyunsaturated Fatty Acids and Allergic Disease," Current Opinion in Clinical Nutrition and Metabolic Care, Vol. 7, No. 2, 2004, pp. 123-129.

[10] J. K. Peat, S. Mihrshahi, A. S. Kemp, G. B. Marks, E. R. 
Tovey, K. Webb, C. M. Mellis and S. R. Leeder, "ThreeYear Outcomes of Dietary Fatty Acid Modification and House Dust Mite Reduction in the Childhood Asthma Prevention Study," The Journal of Allergy and Clinical Immunology, Vol. 114, No.4, 2004, pp. 807-813. doi:10.1016/j.jaci.2004.06.057

[11] T. D. Mickleborough, A. A. Ionescu and K. W. Rundell, "Omega-3 Fatty Acids and Airway Hyperresponsiveness in Asthma," The Journal of Alternative and Complementary Medicine, Vol. 10, No. 6, 2004, pp. 1067-1075. doi:10.1089/acm.2004.10.1067

[12] G. Devereux and Anthony Seaton, "Diet as a Risk Factor for Atopy and Asthma," The Journal of Allergy and Clinical Immunology, Vol. 115, No. 6, 2005, pp. 1109-1117. doi:10.1016/j.jaci.2004.12.1139

[13] K. W. Wong, "Clinical Efficacy of n-3 Fatty Acid Supplementation in Patients with Asthma," Journal of the American Dietetic Association, Vol. 105, No.1, 2005, pp. 98-105. doi:10.1016/j.jada.2004.10.009

[14] C. Almqvist, F. Garden, W. Xuan, S. Mihrshahi, S. R. Leeder, W. Oddy, K. Webb and G. B. Marks, "Omega-3 and Omega-6 Fatty Acid Exposure from Early Life Does Not Affect Atopy and Asthma at Age 5 Years," The Journal of allergy and clinical immunology, Vol. 119, No, 6, 2007, pp. 1438-1444. doi:10.1016/j.jaci.2007.01.046

[15] H. R. Chang, D. Arsenijevic, J. C. Pechère, P. F. Piguet, N. Mensi, L. Girardier and A.G. Dulloo, "Dietary Supplementation with Fish Oil Enhances in Vivo Synthesis of Tumor Necrosis Factor," Immunology Letters, Vol. 34, No. 1, 1992, pp. 13-17. doi:10.1016/0165-2478(92)90021-F

[16] P. S. Tappia, W. J. Man and R. F. Grimble, "Influence of Unsaturated Fatty Acids on the Production of Tumor Necrosis Factor and Interleukin- 6 by Rat Peritoneal Macrophages," Molecular and Cellular Biochemistry, Vol. 143, No. 2, 1995, pp. 89-98. doi:10.1007/BF01816941

[17] D. H. Petursdottir, I. Olafsdottir and I. Hardardottir, "Necrosis Factor Secretion but Decreases Interleukin-10 Secretion by Murine Peritoneal Macrophages," The Journal of Nutrition, Vol. 132, No. 12, 2002, pp. 3740-3743.

[18] A. Pupe, R. Moison, P. De Haes, G. B. van Henegouwen, Lesley Rhodes, H. Degreef and M. Garmyn, "Eicosapentaenoic Acid, a n-3 Polyunsaturated Fatty Acid Differentially Modulates $\mathrm{TNF}_{\alpha}$, IL- $1_{\alpha}$, IL-6 and PGE2 Expression in UVB-Irradiated Human Keratinocytes," The Journal of Investigative Dermatology, Vol. 118, No. 4, 2002, pp. 692-698. doi:10.1046/j.1523-1747.2002.01615.x

[19] M. D. Barber, K. C. H. Fearon and J. A. Ross, "Eicosapentaenoic Acid Modulates the Immune Response but Has No Effect on a Mimic of Antigen-Specific Responses," Ntrition, Vol. 21, No. 5, 2005, pp. 588-593. doi:10.1016/j.nut.2004.09.017

[20] W. C. Chang, C. H. Chen, W. T. K. Cheng and S. T. Ding, "The Effect of Dietary Docosahexaenoic Acid Enrichment on the Expression of Porcine Hepatic Genes," Asian-Australasian Journal of Animal Sciences, Vol. 20, No. 5, 2007, pp. 768-774.

[21] C. H. Chen, P. H. Wang, B. H. Liu, H. H. Hsu, H. J. Mer- smann and S. T. Ding, "Serum Amyloid A Protein Regulates the Expression of Porcine Genes Related to Lipid Metabolism," The Journal of Nutrition, Vol. 138, No. 4, 2008, pp. 674-679.

[22] Y. C. Wang, W. H. Kuo, C. Y. Chen, H. Y. Lin, H. T. Wu, B. H. Liu, C. H. Chen, H. J. Mersmann, K. J. Chang and S. T. Ding, "Docosahexaenoic Acid Regulates Serum Amyloid A Protein to Promote Lipolysis through down Regulation of Perilipin," The Journal of Nutritional Biochemistry, Vol. 21, No. 21, 2010, pp. 317-324. doi:10.1016/j.jnutbio.2009.01.004

[23] P. A. Baeuerle and T. Henkel, "Function and Activation of NF-Kappa B in the Immune System," Annual review of immunology, Vol. 12, No. 1, 1994, pp. 141-179. doi:10.1146/annurev.iy.12.040194.001041

[24] Y. Zhao, S. Joshi-Barve, S.Barve and L. H. Chen, "Eicosapentaenoic Acid Prevents LPS Induced TNF-alpha Expression by Preventing NF-kappaB Activation," Journal of the American College of Nutrition, Vol. 23, No. 1, 2004, pp. 71-78.

[25] S. M. Weldon, A. C. Mullen, C. E. Loscher, L. A. Hurley and H. M. Roche, "Docosahexaenoic Acid Induces an Anti-Inflammatory Profile in Lipopolysaccharide-Stimulated Human THP-1 Macrophages More Effectively than Eicosapentaenoic Acid," The Journal of Nutritional Biochemistry, Vol. 18, No. 4, 2007, pp. 250-258. doi:10.1016/j.jnutbio.2006.04.003

[26] R. Kitz, M. A. Rose, R. Schubert, C. Beermann, A. Kaufmann, H. J. Böhles, J. Schulze and S. Zielen, "Omega-3 Polyunsaturated Fatty Acids and Bronchial Inflammation in Grass Pollen Allergy after Allergen Challenge," Respiratory medicine, Vol. 104, No. 12, 2010, pp. 1793-1798. doi:10.1016/j.rmed.2010.06.019

[27] M. W. Wu, L. J. Xian, X. M. Li, I. Pasquale and L. Francis, "Circadian Expression of Dihydropyrimidine Dehydrogenase, Thymidylate Synthase, c-myc and p53 mRNA in Mouse Livertissue," Ai Zheng, Vol. 23, No. 3, 2004, pp. 235-242.

[28] A. Hirao, H. Nagahama, T. Tsuboi, M. Hirao, Y. Tahara and S. Shibata, "Combination of Starvation Interval and Food Volume Determines the Phase of Liver Circadian Rhythm in Per $2::$ Luc Knock-In Mice under Two Meals Per Day Feeding," American Journal of Physiology. Gastrointestinal and Liver Physiology, Vol. 299, No. 5, 2010, pp. G1045-G1053. doi:10.1152/ajpgi.00330.2010

[29] W. S. Harris, "Fish Oils and Plasma Lipid and Lipoprotein Metabolism in Humans: A Critical Review," The Journal of Lipid Research, Vol. 30, No. 6, 1989, pp. 785-807.

[30] W. S. Harris, "n-3 Fatty Acids and Serum Lipoproteins: Human Studies," American Journal of Clinical Nutrition, Vol. 65, No. 5, 1997, pp. 1645S-1654S.

[31] F. Belzung, T. Raclot and R. Groscolas, "Fish Oil n-3 Fatty Acids Selectively Limit the Hypertrophy of Abdominal Fat Depots in Growing Rats Fed High-Fat Diets," American Journal of Physiology, Vol. 264, No. 6, 1993, pp. R1111-R1118.

[32] J. O. Hill, J. C. Peters, D. Lin, F. Yakubu, H. Greene and 
L. Swift, "Lipid Accumulation and Body Fat Distribution Is Influenced by Type of Dietary Fat Fed to Rats," International Journal of Obesity and Related Metabolic Disorders, Vol. 17, No. 4, 1993, pp. 223-236.

[33] S. Ikemoto, M. Takahashi, N. Tsunoda, K. Maruyama, H. Itakura and O. Ezaki, "High-Fat Diet-Induced Hyperglycemia and Obesity in Mice: Differential Effects of Dietary Oils," Metabolism, Vol. 45, No. 12, 1996, pp. 15391546. doi:10.1016/S0026-0495(96)90185-7

[34] N. Mito, C. Kitada, T. Hosoda and K. Sato, "Effect of Diet-Induced Obesity on Ovalbumin-Specific Immune Res- ponse in a Murine Asthma Model," Metabolism, Vol. 51, No. 10, 2002, pp. 1241-1246. doi:10.1053/meta.2002.35196

[35] H. Yina, W. Liu, K. Goleniewska, N. A. Porter, J. D. Morrow and R. Stokes Peebles Jr., "Dietary Supplementation of Omega-3 Fatty Acid-Containing Fish Oil Suppresses F2-Isoprostanes but Enhances Inflammatory Cytokine Response in a Mouse Model of Ovalbumin-Induced Allergic Lung Inflammation," Free Radical Biology and Medicine, Vol. 47, No. 5, 2009, pp. 622-628. doi:10.1016/j.freeradbiomed.2009.05.033 